\title{
Follow-Up Ileocolonoscopy Is Underused in Crohn's Disease Patients after Ileocecal Resection despite Higher Total and Inpatient Health-Care Costs Compared to Controls
}

\author{
Stephan R. Vavricka ${ }^{a, f}$ Thomas Greuter ${ }^{a}$ e Beat Brüngger ${ }^{b}$ Eva Blozik ${ }^{b}$ \\ Jennifer Celeiro ${ }^{c}$ Alain M. Schoepfer ${ }^{d}$ Caroline Bähler $^{b}$ \\ ${ }^{a}$ Department of Gastroenterology and Hepatology, University Hospital Zurich, Zurich, Switzerland; bepartment \\ of Health Sciences, Helsana Group, Zurich, Switzerland; 'Division of Surgery, Triemli Hospital, Zurich, Switzerland; \\ ${ }^{d}$ Division of Gastroenterology and Hepatology, CHUV - University Hospital Lausanne, Lausanne, Switzerland;

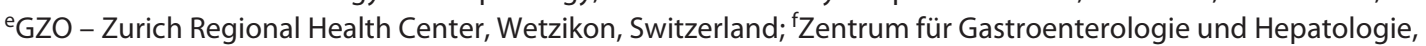 \\ Zurich, Switzerland
}

\section{Keywords}

Crohn's disease - lleocecal resection · Follow-up .

Postoperative recurrence

\begin{abstract}
Background: Postoperative recurrence is frequently observed after ileocecal resection in Crohn's disease (CD) patients. Since 2010, endoscopy within 1 year is considered the gold standard for its diagnosis. However, if and how frequent such endoscopies are performed in clinical practice remains unknown. Methods: We analyzed 1-year follow-up data on $C D$ patients who underwent ileocecal resection between 2012 and 2014 and compared them with hospitalized, non-resected CD controls. Data were extracted from the Helsana database. Helsana is one of the largest Swiss health insurance companies providing coverage for 1.2 million individuals. Results: A total of 645 CD patients were identified with $\geq 1$ hospitalization between 2012 and 2014 and a follow-up of 1 year. Of these, 79 (12.2\%) underwent ileocecal resection. Although endoscopy rates increased over time and were higher in patients with resection versus controls
\end{abstract}

\begin{tabular}{|c|c|}
\hline $\begin{array}{l}\text { karger@karger.com } \\
\text { www.karger.com/iid }\end{array}$ & $\begin{array}{l}\text { (C) } 2020 \text { The Author(s) } \\
\text { Published by S. Karger AG, Basel }\end{array}$ \\
\hline & $\begin{array}{l}\text { This article is licensed under the Creative Commons Attribution- } \\
\text { NonCommercial-NoDerivatives } 4.0 \text { International License (CC BY- } \\
\text { NC-ND) (http://www.karger.com/Services/OpenAccessLicense). } \\
\text { Usage and distribution for commercial purposes as well as any dis- } \\
\text { tribution of modified material requires written permission. }\end{array}$ \\
\hline
\end{tabular}

$(p=0.029)$, in only $54.4 \%$ a 1 -year follow-up ileocolonoscopy was performed. Postoperative prophylaxis with anti-tumor necrosis factor or azathioprine was prescribed in $63.3 \%$. Female sex and age $>60$ years were independent predictors for not receiving prophylaxis (odds ratio [OR] $0.36, p=0.048$, and OR $0.2, p=0.022$ ). Patients with resection had significantly lower numbers of rehospitalizations (1.2 vs. 1.8, $p=$ 0.021 ), with resection being an independent negative predictor for number of rehospitalizations in a Poisson regression model (incident risk ratio $0.64, p=0.029$ ). However, disease-related surgery was more often the cause for rehospitalization after resection versus controls ( 47.6 vs. $22.1 \%, p=$ $0.015)$. Total and inpatient health-care costs were higher in these patients. Conclusion: Endoscopies are underused after ileocecal resection. This contrasts current guidelines. Physicians should be aware of this underuse and perform follow-up examinations more often.

(C) 2020 The Author(s)

Published by S. Karger AG, Basel

Stephan R. Vavricka and Thomas Greuter share co-first authorship. Alain M. Schoepfer and Caroline Bähler share last authorship.
Prof. Stephan R. Vavricka

Division of Gastroenterology and Hepatology University Hospital Zurich

Rämistrasse 100, Zürich 8091 (Switzerland)

stephan.vavricka@usz.ch 


\section{Introduction}

Due to its chronic nature, Crohn's disease (CD) usually requires medical maintenance treatment after an initial induction phase. Biological agents, such as anti-tumor necrosis factor (TNF) and more recently anti-integrins (vedolizumab), have changed long-term management of $\mathrm{CD}$ in the last decade with higher response rates than before with conventional immunosuppressive therapies [1-3]. However, surgical treatment is still needed in a considerable proportion of patients. Indeed, in patients presenting with ileocecal CD, there is a $90 \%$ likelihood of requiring surgery, while recurrent inflammation requiring another resection affects every second patient [4-8]. Ileocecal resection with an ileocolic side-to-side anastomosis - usually performed laparoscopically - is the preferred option in patients with isolated ileoecal CD with obstructive symptoms, but absence of active inflammation. In addition, ileocecal resection is needed in case of refractory obstruction despite initial medical treatment [9].

Although short-term morbidity and mortality of ileocecal resection are very low if performed at experienced large-volume tertiary centers [10], postoperative recurrence (POR) of CD is a frequently observed problem. Several risk factors have been identified, among which are current smoking, prior intestinal surgery, absence of prophylactic treatment, penetrating disease at index surgery, and perianal disease [9]. Clinical POR has been reported for $28-45 \%$ at 5 years and $36-61 \%$ at 10 years. However, histological or endoscopic recurrence precedes such clinical POR and may occur within weeks to months after index surgery [11-16]. Indeed, symptom scores such as the Crohn's disease activity index (CDAI) have a poor sensitivity [17]. In contrast, endoscopic disease activity best predicts postoperative clinical course, and treatment adjustment based on regular colonoscopies 6 months after surgery has been demonstrated to be more effective than conventional management in a randomized controlled trial $[14,18,19]$. Since 2010, the European Crohn's and Colitis Organization (ECCO) guidelines recommend ileocolonoscopy within the first year after ileocecal resection as the gold standard for the diagnosis of POR. Furthermore, early prophylactic treatment with either thiopurines or anti-TNF should be considered, if 1 or more risk factors for POR are present [9].

The recently published and comprehensive Practicrohn study from 26 centers in Spain evaluated 314 patients who underwent ileocecal resection and demonstrated a prophylaxis rate of $68 \%$ and a 1 -year follow-up endoscopy rate of less than 50\% [20]. At that time (20072010), the aforementioned ECCO guidelines had not been introduced, assuming higher rates in current clinical practice. However, whether follow-up endoscopies are now performed indeed more often remains unknown.

Given this lack of knowledge regarding current adherence rates to the ECCO guidelines, we analyzed 1-year follow-up in patients who underwent ileocecal resection between 2012 and 2014 and compared them with hospitalized CD patients without such resection in a large Swiss $\mathrm{CD}$ cohort. We aimed at obtaining a more up-to-date picture of current postoperative management in CD patients after ileocecal surgery by answering the following questions: (1) How often are follow-up endoscopies performed after resection, and are these patients followed up more closely than controls? (2) How are patients treated after ileocecal resection, and is there a difference from non-resected controls? (3) How often are patients rehospitalized compared to controls, and does this have an impact on health-care costs?

\section{Methods}

Study Design

We performed an observational study analyzing a large Swiss cohort of CD patients. All data were extracted from the anonymized patient information database of Helsana, one of the largest Swiss health insurance companies providing health-care coverage for approximately $15 \%$ of the Swiss population corresponding to a total of 1.2 million insured individuals. All requirements of the article 22 of the Swiss data protection law were fulfilled, which permits the use of insurance data for research purposes in this study. Therefore, this study was exempted from Ethics Committee approval.

\section{Study Population and Data Collection}

All CD patients included in the Helsana database were identified by a keyword search using the International Classification of Diseases (ICD)-10, revision code K50, by means of the DRG ("diagnosis-related group") system, which was introduced for the stationary setting in Switzerland in 2012. Consistent diagnostic coding in the ambulatory setting was based on the tariff system Tarmed, which was introduced in Switzerland and implemented at Helsana in 2004 [21]. Demographic information, items related to medication use, surgery, hospitalization, and health-care costs were retrieved from the Helsana database as a result of this keyword search. All CD patients with at least 1 hospitalization during the period of 2012-2014 were included for further analysis. The exclusion criteria were (i) missing follow-up data in the first year after index surgery, which is the case for Helsana employees, asylum seekers, nursing home residents whose medical costs were covered by a fixed rate - the reason why no detailed information on the type of medication was available - and individuals living abroad; (ii) age < 18 years; (iii) death during the follow-up period 2012-2014; and (iv) loss to follow-up during the period 2012-2014 (e.g., dropout from Helsana coverage). 
Table 1. Baseline characteristics of included patients $(n=645)$

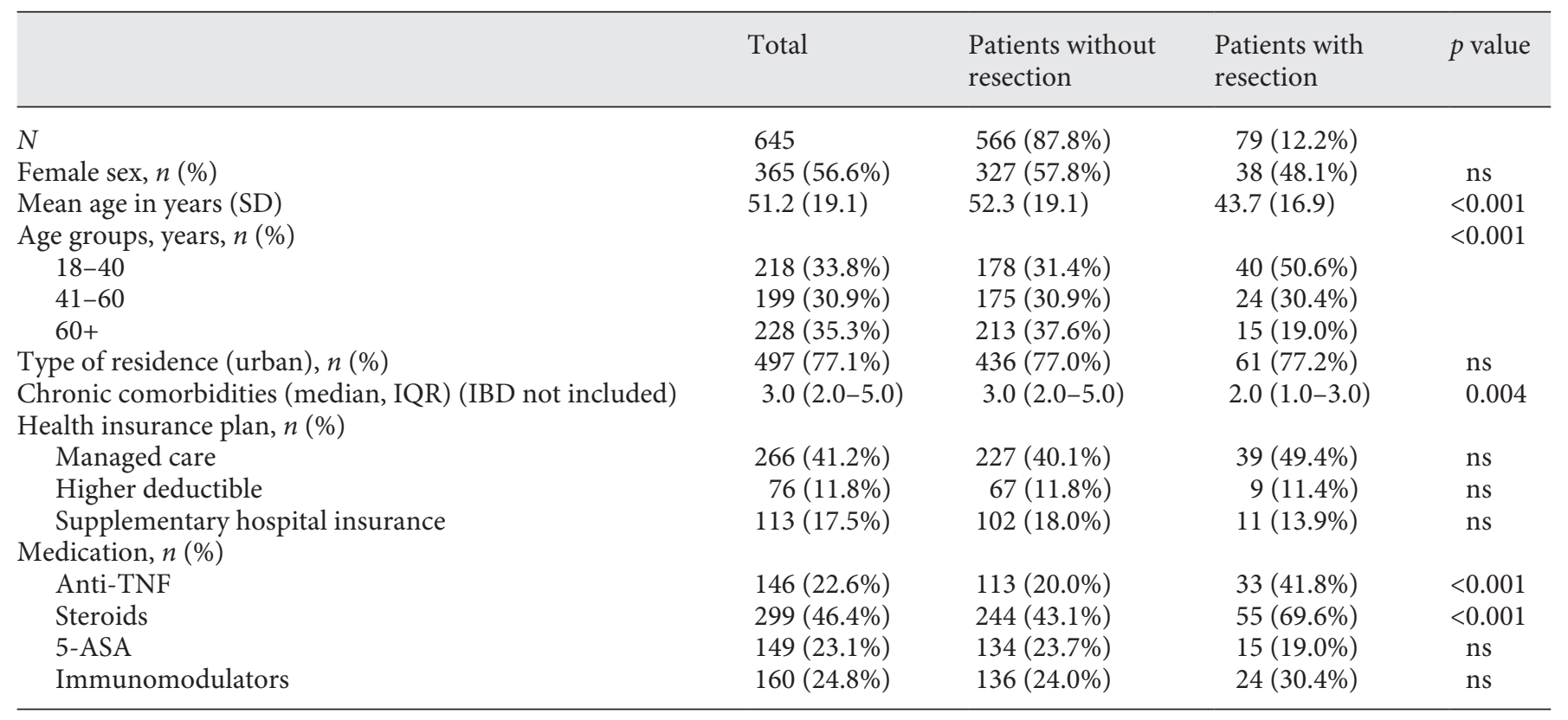

$p$ values are calculated using Fisher's exact test for dichotomous variables, the Kruskal-Wallis test for continuous variables, and the $\chi^{2}$ test for categorical variables. ns, not significant; TNF, tumor necrosis factor; 5-ASA, 5-aminosalicylic acid; IQR, interquartile range; IBD, inflammatory bowel disease; SD, standard deviation.

\section{Outcome Measures}

The following set of variables was extracted from the Helsana database for further analyses: sex; age-group (18-40, 41-60, and $60+$ years); number of chronic medical conditions $(0-1,2-4$, or $5+$ ); type of insurance coverage (managed care model, deductible class, and supplementary hospital insurance); type of residence (urban vs. rural); type of CD medication (5-aminosalicylic acid; immunosuppressive agent; biologics, particularly anti-TNF; steroids; and antibiotics such as metronidazole); number and frequency of rehospitalizations (any hospitalization), including reasons for rehospitalization (such as surgery in general and diseaserelated surgery); follow-up examinations within 1 year after index surgery (magnetic resonance imaging, computed tomography, Xray, sonography, fecal calprotectin, and ileocolonoscopy); frequency, number, and type of follow-up visits; total, outpatient, and inpatient costs (in Swiss Francs); and time point of re-initiation of CD medication after index surgery/hospitalization. For translation of costs in USD, a currency rate of $1 \mathrm{CHF}=1.02$ USD was used.

\section{Statistical Analysis}

For all statistical analyses, R statistics, version 3.2.0., was used. Data distribution was analyzed using Normal-QQ-Plots. Results of quantitative data are presented as either mean \pm SD (for normally distributed data) or median plus interquartile range (in case of non-normal distribution). Categorical data were summarized as the percentage of the group total. For comparisons between continuous variables, the Kruskal-Wallis test was used. Comparison between categorical data was performed using the $\chi^{2}$ test, while for dichotomous variables Fisher's exact test was used. Poisson regression modeling was performed to analyze the association between number of rehospitalizations and the presence of ileocecal resection, linear regression modeling was used to investigate the association between health-care costs (total, inpatient, and outpatient) and the presence of ileocecal resection, and multivariate logistic regression analysis for the assessment of factors associated with postoperative prophylaxis and adequate postoperative follow-up examinations. The following influencing factors (independent variables) were included into the models to adjust for potential confounders: age group (18-40, 41-60, and 60+ years), sex, number of chronic medical conditions ( $0-1,2-4$, and $5+)$, and type of health insurance coverage. In the presence of an obviously skewed distribution of the cost data, log transformation was used. For calculation of time to re-initiation of CD medication after index surgery/hospitalization, a Kaplan-Meier analysis was performed. Kaplan-Meier curves were statistically compared using Cox proportional hazard ratios. A two-sided $p$ value of $<0.05$ was regarded as statistically significant.

\section{Results}

\section{Patient Demographics}

We identified 762 CD patients with at least 1 hospitalization between 2012 and 2014. Of those, 20 (2.6\%) were excluded due to missing data, 21 (2.8\%) due to age below 18 years, $44(5.8 \%)$ because of death within the follow-up period, and $32(4.2 \%)$ due to loss to follow-up. The final sample comprised 645 adult CD patients with at least 1 
Fig. 1. Aftercare in patients with (a) and without resection (b) in the 1-year followup.

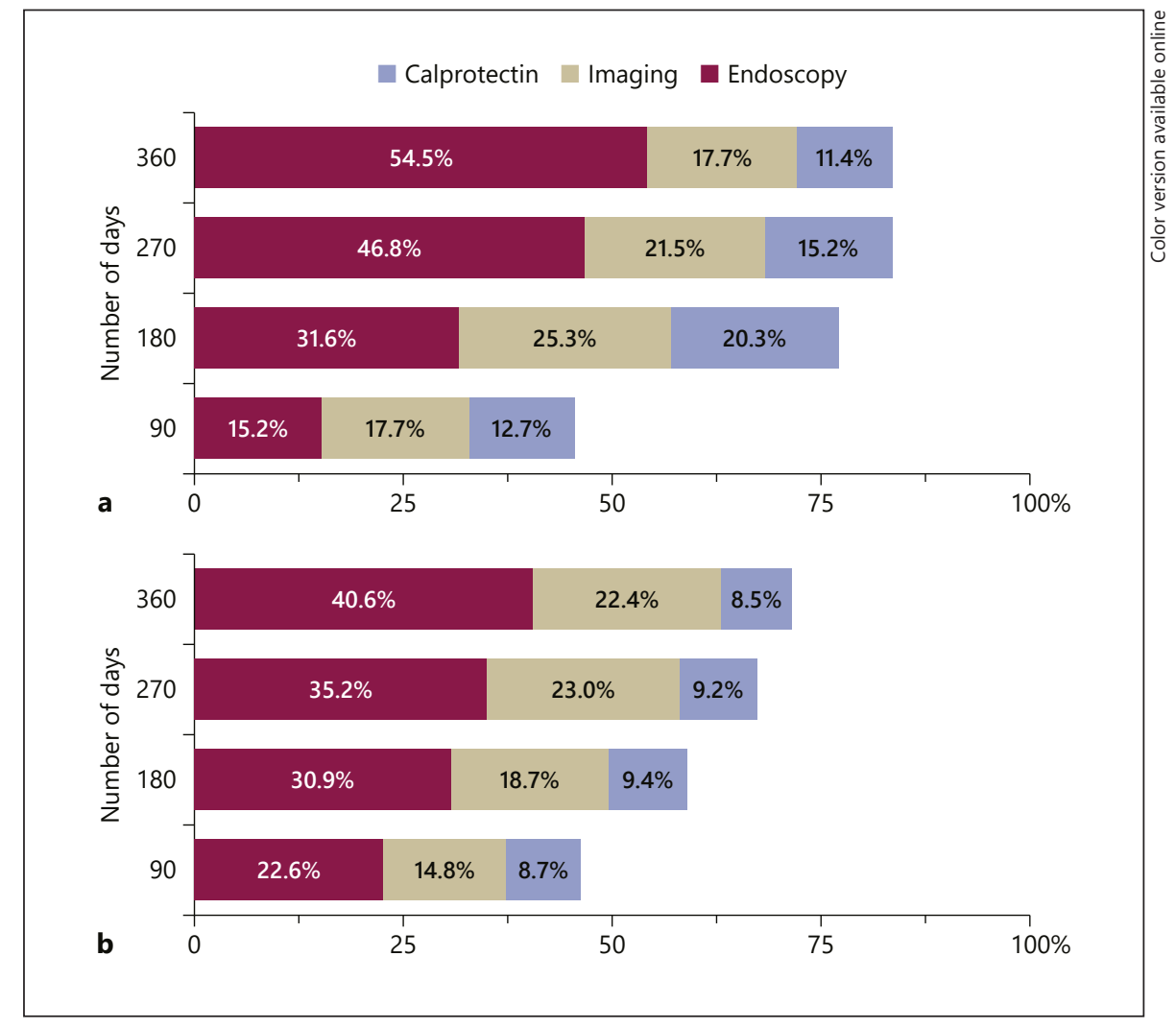

hospitalization between 2012 and 2014 and a follow-up of 1 year. Of those, 79 (12.2\%) underwent an ileocecal resection (mean age 43.7 years, $48.1 \%$ females). Table 1 shows the demographic data of included patients at baseline.

\section{Follow-Up Evaluations}

Although endoscopy rates increased over time after ileocecal resection and endoscopies were more frequently performed in patients with resection versus patients without ( 54.4 vs. $40.6 \%, p=0.029$ ), 1 year after index surgery, follow-up ileocolonoscopy was missing in $45.6 \%$. Even when taking alternative diagnostic tools into account such as imaging or fecal calprotectin, $16.5 \%$ of the patients in the resection group did not receive any follow-up examination. Figure 1 shows different follow-up examinations over time after the index surgery/hospitalization according to whether patients had ileocecal resection or not. After ileocecal resection, patients were mostly followed up not only by their gastroenterologists (number of visits 10.0 ), but also primary care physicians ( 7.0 visits) and other specialists (6.0 visits). No difference regarding sex, age, type of residence, chronic conditions, or type of health insurance was seen when patients with an adequate follow-up after ileocecal resection were compared

Follow-Up of Crohn's Disease Patients after Ileocecal Resection with patients who did not receive such follow-up (see online suppl. Table 1; for all online suppl. material, see www. karger.com/doi/10.1159/000507115). No predictors for receiving adequate follow-up versus not were identified.

\section{Medications}

Patients with ileocecal resection were frequently treated with biologics (particularly anti-TNF), but not steroids, in the postoperative setting. Rates of biological treatment were higher than in patients without ileocecal resection (for biologics, 49.4 vs. $29.7 \%, p<0.001$, and for anti-TNF, 48.1 vs. $29.5 \%, p=0.001$ ), while those for steroids were lower ( 39.2 vs. $54.9 \%, p=0.011$ ). Since steroid use has been associated with higher rates of postoperative complications and data on anti-TNF and infectious complications after $\mathrm{CD}$ surgery are still conflicting, we sought to analyze the time from index surgery to first dose of steroids and anti-TNF, respectively. In patients who were treated with steroids and/or anti-TNF before surgery, first postoperative application was significantly delayed when compared with patients without ileocecal resection $(p<$ 0.001 and $p=0.011$ ). No such delayed introduction was reported for azathioprine. For details, see the Kaplan-Meier curves in Figures 2 and 3, and online suppl. Figure 1. 


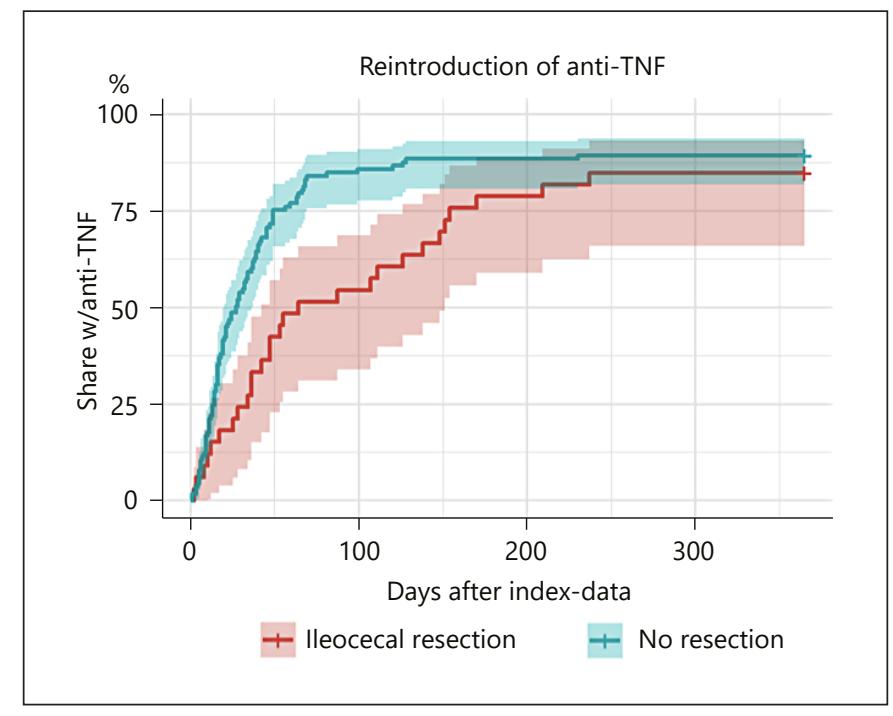

Fig. 2. Kaplan-Meier curves showing reinitiation of anti-TNF in the 1-year follow-up. TNF, tumor necrosis factor.

\section{Postoperative Prophylaxis}

Prophylaxis for recurrence of CD after ileocecal resection was initiated in 50 of the 79 patients $(63.3 \%)$, while $29(36.7 \%)$ did not receive any prophylactic treatment (anti-TNF or azathioprine). Metronidazole was prescribed in $22.8 \%$ of patients. The proportion of females was significantly less in the patient group that received prophylactic medication ( 38.0 vs. $65.5 \%, p=0.022$ ). Indeed, female sex was an independent prognostic factor for not receiving postoperative prophylactic treatment even after controlling for age and further chronic conditions or urbanity (odds ratio [OR] $0.36, p=0.048$ ). In addition, patients aged 61 years or older were more likely not treated with anti-TNF or azathioprine than patients aged 40 years and younger (OR $0.20, p=0.022$ ). For the multivariate analysis, see Table 2 . Otherwise, the 2 groups did not show any differences (online suppl. Table 2).

\section{Rehospitalizations}

CD patients with resection were frequently readmitted to the hospital, although there was a trend toward a lower frequency of rehospitalization than in patients without ileocecal resection ( 26.6 vs. $37.6 \%, p=0.061)$. In fact, the number of rehospitalizations was lower after ileocecal resection ( 1.2 vs. $1.8, p=0.021$, Table 3 ). Ileocecal resection was an independent negative predictor for number of rehospitalizations in a Poisson regression model corrected for age, sex, and chronic medical conditions (incident risk ratio $0.64, p=0.029$, Table 4). However, disease-related

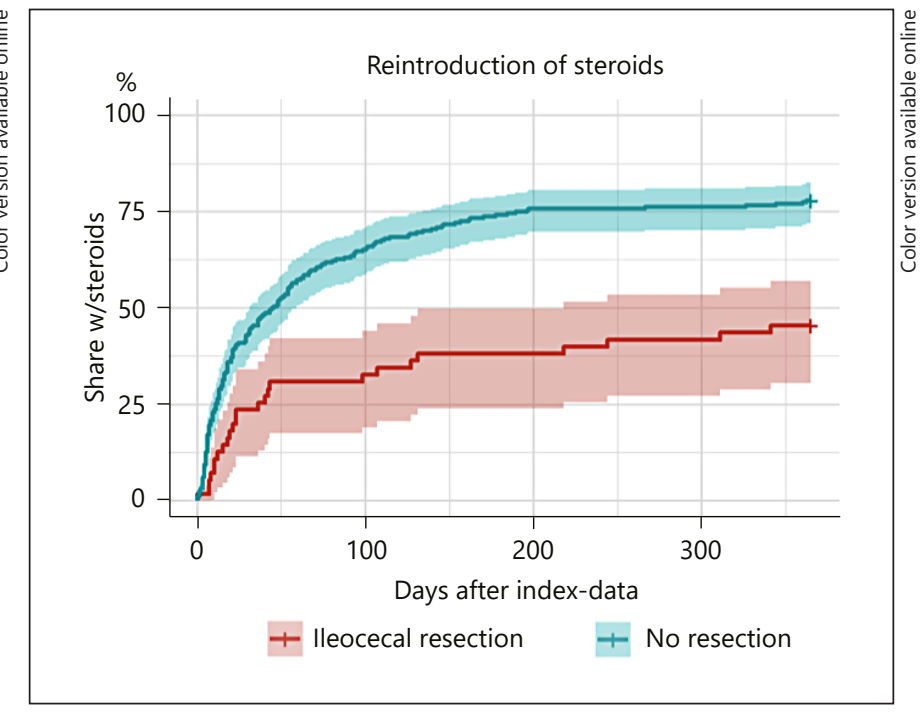

Fig. 3. Kaplan-Meier curves showing reinitiation of steroids in the 1-year follow-up.

Table 2. Multivariate regression model for prediction of receiving postoperative prophylactic treatment with anti-TNF and/or azathioprine

\begin{tabular}{llll}
\hline $\begin{array}{l}\text { Prophylactic treatment with } \\
\text { anti-TNF and/or azathioprine }\end{array}$ & OR & 95\% CI & $p$ value \\
\hline Female sex & 0.36 & $0.13-0.98$ & 0.048 \\
Age 18-40 years & 1.00 & & \\
Age 41-60 years & 0.81 & $0.25-2.64$ & 0.721 \\
Age 60+ years & 0.20 & $0.05-0.76$ & 0.022 \\
Type of residence (urban) & 1.31 & $0.37-4.48$ & 0.669 \\
2-4 chronic conditions & 1.79 & $0.56-5.79$ & 0.321 \\
5+ chronic conditions & 1.850 & $0.31-13.51$ & 0.515 \\
\hline
\end{tabular}

TNF, tumor necrosis factor; OR, odds ratio; CI, confidence interval.

surgery was more often the cause for rehospitalization in patients after resection than in those without (47.6 vs. $22.1 \%, p=0.015)$.

\section{Health-Care Costs}

Health-care costs in the 1-year follow-up were significantly higher in patients with resection than in patients without ( 28,340 vs. $23,480 \mathrm{CHF}, 28,907$ vs. $23,950 \mathrm{USD}$, $p=0.020)$, mainly due to inpatient (11,290 vs. $5,920 \mathrm{CHF}$, $11,516$ vs. 6,038 USD, $p<0.001)$ but not outpatient costs including medications and laboratory testing $(13,200$ vs. 12,710 CHF, 13,464 vs. 12,964 USD, ns) (for details, see 
Table 3. Rehospitalizations and surgery after index hospitalization

\begin{tabular}{lclll}
\hline & Total & $\begin{array}{l}\text { Patients without } \\
\text { resection }\end{array}$ & $\begin{array}{l}\text { Patients with } \\
\text { resection }\end{array}$ & $p$ value $^{\mathrm{a}}$ \\
$N$ & 645 & $566(87.8 \%)$ & $79(12.2 \%)$ & 0.061 \\
Rehospitalization, $n(\%)_{\text {Rehospitalizations }}^{\mathrm{b}}$ (mean, SD), $n$ & $234(36.3 \%)$ & $213(37.6 \%)$ & $21(26.6 \%)$ & 0.021 \\
Surgery $^{\mathrm{b}}, n(\%)$ & $1.7,1.2$ & $1.8,1.2$ & $1.2,0.5$ & $14(66.7 \%)$ \\
Disease-related surgery $^{\mathrm{b}}, n(\%)$ & $129(55.1 \%)$ & $115(54.0 \%)$ & $10(47.6 \%)$ & 0.015 \\
\hline
\end{tabular}

Ns, not significant; SD, standard deviation. ${ }^{a} p$ values were calculated using Fisher's exact test for dichotomous variables and the Kruskal-Wallis test for continuous variables. ${ }^{\mathrm{b}}$ In those patients with at least one rehospitalization.

Table 4. Poisson regression model evaluating factors for number of rehospitalizations during the 1-year follow-up period

\begin{tabular}{lccc}
\hline Number of rehospitalizations & IRR & $95 \%$ CI & $p$ value \\
\hline CD with resection & 0.64 & $0.42-0.94$ & 0.029 \\
Age 18-40 years & 1.00 & & \\
Age 41-60 years & 1.23 & $0.93-1.64$ & $\mathrm{~ns}$ \\
Age 60+ years & 1.26 & $0.95-1.68$ & $\mathrm{~ns}$ \\
Female sex & 0.93 & $0.76-1.14$ & $\mathrm{~ns}$ \\
2-4 chronic conditions & 2.79 & $1.89-4.27$ & $<0.001$ \\
5+ chronic conditions & 5.48 & $3.68-8.46$ & $<0.001$ \\
\hline
\end{tabular}

IRR, incident risk ratio; CD, Crohn's disease; CI, confidence interval; ns, not significant.

Table 5). In a linear regression model corrected for age, sex, and number of additional chronic medical conditions, ileocecal resection was an independent predictor for increased total and inpatient health-care costs (OR $1.40, p<0.001$ and OR 2.17, $p<0.001$ ). For details regarding regression analyses, see online suppl. Table 3 (total costs) and online suppl. Table 4 (inpatient costs).

\section{Discussion}

Due to high rates of postoperative disease recurrence after ileocecal resection, early endoscopy within 1 year after surgery is recommended according to current ECCO guidelines. Recent studies have implicated increasing frequency of follow-up endoscopies; however, they were performed before the introduction of these guidelines. Knowledge about current postoperative management and adherence to these guidelines is missing. Therefore, we performed a retrospective analysis of the 1-year follow-up of patients who underwent ileocecal resection between 2012 and 2014 and compared it with the follow-up of a control group of hospitalized but non-resected CD patients. Thereby we aimed to obtain an up-to-date picture of the current postoperative management in CD patients and of their disease course after ileocecal resection.

With a 1 -year rate of only $54 \%$, follow-up endoscopies are underused in the postoperative setting. This finding considerably contrasts current guidelines on postoperative follow-up examinations after ileocecal resection [9]. Ileocolonoscopy is considered the gold standard in the diagnosis of POR and is recommended within the first year after surgery. The rationale is based on 2 facts: (1) POR is frequent with a cumulative incidence of $28-45 \%$ after 5 years and $36-61 \%$ after 10 years, and (2) the postoperative clinical course of $\mathrm{CD}$ is best predicted by the severity of endoscopic lesions, while CDAI has a poor correlation with endoscopic disease activity and POR [9, $14,22]$. Indeed, histologic and endoscopic recurrence may occur within even few weeks to months after surgery, and the use of follow-up endoscopies has been demonstrated to be efficacious [19]. Regueiro and colleagues [17] reported on a 1-year endoscopic recurrence rate of $50 \%$. Of note, the rate of follow-up endoscopies in our cohort did not significantly differ from that reported in the recently published Practicrohn study, which included the follow-up of 314 patients after ileocecal resection (143/314 with 1 -year endoscopy vs. 43/79 in our cohort, $p=0.157$ ) [20]. This is even more astonishing given the fact that the current guidelines have not been implemented at the time when surgeries were performed in that study (2007-2010). Domènech et al. [20], however, clearly showed increasing rates of 1-year endoscopy over time, with an increase from $33.8 \%$ in 2007 to $59.5 \%$ in 2010 . 
Table 5. Total, inpatient, and outpatient costs (in Swiss Francs) during the 1-year follow-up

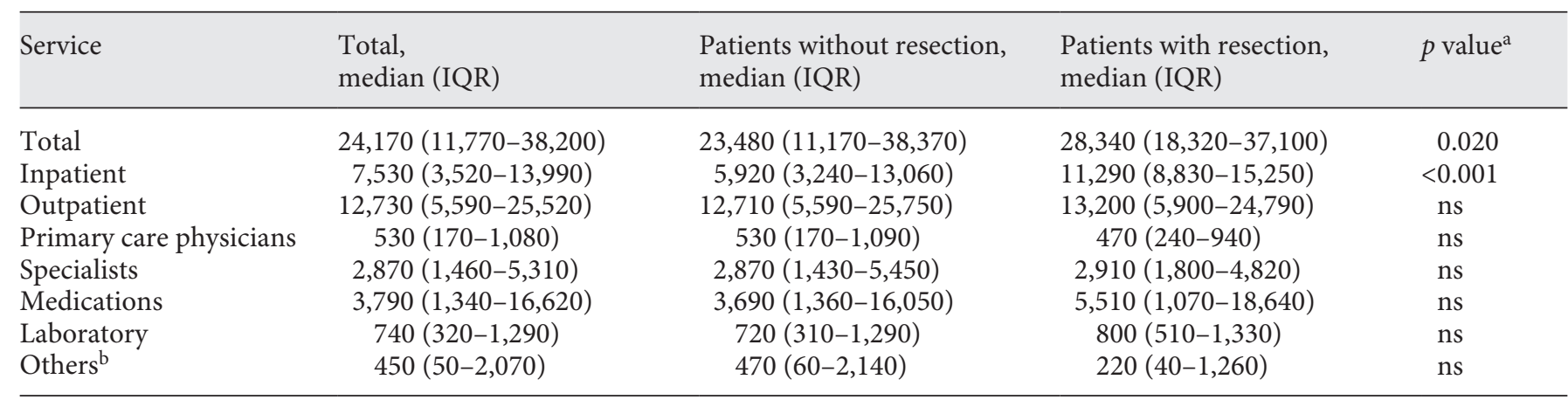

${ }^{\mathrm{a}} p$ values were assessed using the Kruskal-Wallis test. ${ }^{\mathrm{b}}$ Costs incurred by paramedical visits (e.g., physiotherapists), home care nursing services, and use of medical devices. IQR, interquartile range; ns, not significant.

With regard to this, no further increase was observed in our study and the rate seemed to have somewhat plateaued. The rate of follow-up examinations is higher, if alternative methods (evidence level III according to the Oxford Centre for Evidence-Based Medicine) such as measurement of calprotectin and imaging are taken into account [23-27], but there still is a considerable proportion of patients without any of such follow-ups (almost $20 \%)$.

Anti-TNF and steroids were reintroduced after the index surgery with a significant delay compared to controls after index hospitalization. In addition, patients after surgery were less likely to be treated with steroids than control CD patients. Delayed reintroduction was not observed with azathioprines. These findings are in consistence with current evidence and guidelines regarding these drugs. Azathioprine is considered safe and does not increase surgical complications $[28,29]$. Therefore, a delayed application would not make any sense. In contrast, data on anti-TNF are rather controversial, and a recent meta-analysis has demonstrated a trend toward higher rates of total complications with a modestly increased risk of infections, mostly remote from surgical sites [30, 31]. Steroids are clearly associated with higher rates of surgical complications, particularly when taken at a dose of $\geq 20$ mg for more than 6 weeks $[9,28]$. These drugs should, therefore, not be prescribed within the first weeks after surgery.

In our cohort, 50 of 79 patients $(63.3 \%)$ received postoperative prophylaxis with either anti-TNF or azathioprine. This rate is comparable to the one reported by Domènech and colleagues [20] (68\%). While we did not assess for the known predictors of POR [9] in detail due to the retrospective nature of the study and the limited data on these factors in the Helsana database, we found that both female sex and advanced age ( $>60$ years) were negative predictors for receiving a postoperative prophylaxis. This contrasts current guidelines, which clearly state that neither age nor sex is significantly associated with a negative outcome after surgery [9]. No such difference has further been reported in the Practicrohn study [20]. Whether this gender-specific difference in the prescription of prophylactic medication is true or biased by the presence of confounders (such as differences in disease severity or smoking rates) has to be proven in a further study.

Despite the underuse of follow-up examinations, total health-care costs were higher in patients after ileocecal resection than controls, which is mainly due to higher inpatient costs. The rate of rehospitalization after surgery is comparable with the rate reported by Domènech [20] and colleagues (26.6 vs. $18 \%$ ), with POR and surgery-related complications being the most frequent causes for hospitalization. Such increased costs due to postoperative recurrence and disease-related surgery leading to rehospitalizations might be preventable by follow-up examinations as recommended in current guidelines. Awareness of this considerable and impactful underuse should prompt physicians to follow the guidelines more rigorously and recommend patients a close follow-up including endoscopic disease assessment. This might have a positive effect on both costs and hospitalization rates. It however has yet to be determined whether doctors underuse endoscopies because they are unaware of current recommendations or if there is a proportion of physicians who are not convinced by the current evidence that their patients clinically benefit from such an approach. 
Our study has several strengths, but certain limitations as well. According to our knowledge, this is the first study that systematically assessed 1-year follow-up of patients after ileocecal resection after the introduction of the ECCO guidelines with the clear recommendation of ileocolonoscopy within the first year (in 2010). Data collection and analysis relied on unambiguous ICD codes, which makes it possible to include all hospitalized CD patients regardless of the hospital level. Thereby, data analysis is not biased toward tertiary, inflammatory bowel disease referral centers preselecting a specific group of $\mathrm{CD}$ patients. ICD codes have been further demonstrated to be an adequate approach to identify and select patients [32]. Nevertheless, our CD cohort is not entirely representative for the Swiss inflammatory bowel disease population, since Helsana only covers 15\% of the Swiss inhabitants. Furthermore, the inclusion of hospitalized patients only might have led to a certain selection bias toward patients with $C D$ with a greater disease extent. A clear limitation is the fact that we could not include risk factors for early POR (such as smoking behavior, initial disease extent, and disease severity such as perianal disease or penetrating disease course) in our analysis, since these were not reported in the Helsana database. To calculate readmission rates in the postoperative setting, we included all cases with rehospitalization regardless of the underlying cause for admission (7 of 22 readmissions were not related to the gastrointestinal tract). However, we further stratified our results by reason for hospitalization.

Taken together, follow-up endoscopies are not performed in a considerable proportion of patients despite clear recommendations by the ECCO guidelines, current evidence for a benefit from such a strategy, and readily available health-care resources. Fecal calprotectin and cross-sectional imaging are used as an alternative at least in some patients, although evidence for their efficacy is only modest. Treating physicians should be aware of this underuse, and further studies regarding alternative strategies for detection of POR are needed.

\section{Acknowledgements}

The authors thank Sonja Wehrle, Sonja Aerne, and Mikaël Thomas for their helpful support in coding inpatient and outpatient procedures.

\section{Statement of Ethics}

All requirements of the article 22 of the Swiss data protection law were fulfilled, which permits the use of insurance data for research purposes in this study. Therefore, this study was exempted from Ethics Committee approval.

\section{Disclosure Statement}

A.M.S. consulted and received speaker's honoraria from AbbVie, UCB, Falk, Merck Sharp \& Dohme AG (MSD), Tillotts, Vifor, Pfizer, Ferring, Receptos, and Takeda. T.G. consulted and received travel grants from Atlantic Healthcare, Falk, Sanofi, and Vifor. S.R.V. consulted and received speaker's honoraria from AbbVie, UCB, Falk, MSD, Tillotts, Vifor, Pfizer, Takeda, and Ferring. The other authors have no competing interests to declare.

\section{Funding Sources}

This study was funded by MSD (Switzerland). The sponsor reviewed, discussed, and approved the protocol CH-LCE-6247/2017ms-6461. Conduction, analysis, and interpretation of the data, and preparation and submission of the manuscript were solely the responsibility of the authors, without any obligations to the sponsor.

\section{Author Contributions}

S.R.V. and T.G. take responsibility for the integrity of the work as a whole, from inception to the published article. Author contributions: Study conception: A.M.S., C.B., S.R.V., and T.G.; data collection: B.B., C.B., and EB; statistical analysis: B.B., C.B., and E.B.; interpretation of data: A.M.S., C.B., S.R.V., and T.G.; drafting of the manuscript: A.M.S., S.R.V., and T.G.; and critical review of the manuscript: B.B., C.B., E.B., and J.C. All authors approved the final version of the manuscript.

\section{References}

1 Hanauer SB, Feagan BG, Lichtenstein GR, Mayer LF, Schreiber S, Colombel JF, et al. Maintenance infliximab for Crohn's disease: the ACCENT I randomised trial. Lancet. 2002;359(9317):1541-9.

2 Sandborn WJ, Feagan BG, Rutgeerts P, Hanauer S, Colombel JF, Sands BE, et al. Vedolizumab as induction and maintenance therapy for Crohn's disease. N Engl J Med. 2013;369(8):711-21.
3 Colombel JF, Sandborn WJ, Reinisch W, Mantzaris GJ, Kornbluth A, Rachmilewitz D, et al. Infliximab, azathioprine, or combination therapy for Crohn's disease. N Engl J Med. 2010;362(15):1383-95.

4 Cullen G, O'toole A, Keegan D, Sheahan K, Hyland JM, O'donoghue DP. Long-term clinical results of ileocecal resection for Crohn's disease. Inflamm Bowel Dis. 2007;13(11): 1369-73.
5 Shivananda S, Hordijk ML, Pena AS, Mayberry JF. Crohn's disease: risk of recurrence and reoperation in a defined population. Gut. 1989;30(7):990-5.

6 Farmer RG, Whelan G, Fazio VW. Long-term follow-up of patients with Crohn's disease. Relationship between the clinical pattern and prognosis. Gastroenterology. 1985;88(6): 1818-25.
Follow-Up of Crohn's Disease Patients after Ileocecal Resection
Inflamm Intest Dis 2020;5:100-108 DOI: $10.1159 / 000507115$ 
7 Bernell O, Lapidus A, Hellers G. Risk factors for surgery and postoperative recurrence in Crohn's disease. Ann Surg. 2000;231(1):3845.

8 Bemelman WA, Allez M. The surgical intervention: earlier or never? Best Pract Res Clin Gastroenterol. 2014;28(3):497-503.

9 Gionchetti P, Dignass A, Danese S, Magro Dias FJ, Rogler G, Lakatos PL, et al. 3rd European evidence-based consensus on the diagnosis and management of Crohn's disease 2016: part 2: surgical management and special situations. J Crohns Colitis. 2017;11(2):135-49.

10 Soop M, Larson DW, Malireddy K, Cima RR, Young-Fadok TM, Dozois EJ. Safety, feasibility, and short-term outcomes of laparoscopically assisted primary ileocolic resection for Crohn's disease. Surg Endosc. 2009; 23(8):1876-81.

11 Rutgeerts P, Geboes K, Vantrappen G, Kerremans R, Coenegrachts JL, Coremans G. Natural history of recurrent Crohn's disease at the ileocolonic anastomosis after curative surgery. Gut. 1984;25(6):665-72.

12 Whelan G, Farmer RG, Fazio VW, Goormastic M. Recurrence after surgery in Crohn's disease. Relationship to location of disease (clinical pattern) and surgical indication. Gastroenterology. 1985;88(6):1826-33.

13 Tytgat GN, Mulder CJ, Brummelkamp WH. Endoscopic lesions in Crohn's disease early after ileocecal resection. Endoscopy. 1988; 20(5):260-2.

14 Rutgeerts P, Geboes K, Vantrappen G, Beyls J, Kerremans R, Hiele M. Predictability of the postoperative course of Crohn's disease. Gastroenterology. 1990;99(4):956-63.

15 Olaison G, Smedh K, Sjödahl R. Natural course of Crohn's disease after ileocolic resection: endoscopically visualised ileal ulcers preceding symptoms. Gut. 1992;33(3):331-5.

16 Caprilli R, Andreoli A, Capurso L, Corrao G, D’Albasio G, Gioieni A, et al. Oral mesalazine (5-aminosalicylic acid; asacol) for the prevention of post-operative recurrence of Crohn's disease. Gruppo Italiano per lo Studio del Colon e del Retto (GISC). Aliment Pharmacol Ther. 1994;8(1):35-43.

17 Regueiro M, Kip KE, Schraut W, Baidoo L, Sepulveda AR, Pesci M, et al. Crohn's disease activity index does not correlate with endoscopic recurrence one year after ileocolonic resection. Inflamm Bowel Dis. 2011; 17(1):118-26.

18 Ananthakrishnan AN. Surgery for Crohn's disease: look harder, act faster. Lancet. 2015, 385(9976):1370-1.

19 De Cruz P, Kamm MA, Hamilton AL, Ritchie KJ, Krejany EO, Gorelik A, et al. Crohn's disease management after intestinal resection: a randomised trial. Lancet. 2015 385(9976):1406-17.

20 Domènech E, Garcia V, Iborra $\mathrm{M}$, Gutiérrez $\mathrm{A}$, García-López S, Martín Arranz MD, et al. Incidence and management of recurrence in patients with Crohn's disease who have undergone intestinal resection: the practicrohn study. Inflamm Bowel Dis. 2017;23(10):1840-6.

21 Vavricka SR, Schoepfer AM, Safroneeva E, Rogler G, Schwenkglenks M, Achermann R. A shift from oral to intravenous iron supplementation therapy is observed over time in a large swiss cohort of patients with inflammatory bowel disease. Inflamm Bowel Dis. 2013; 19(4):840-6.

22 Vuitton L, Koch S, Peyrin-Biroulet L. Preventing postoperative recurrence in Crohn's disease: what does the future hold? Drugs. 2013;73(16):1749-59.

23 Schoepfer AM, Lewis JD. Serial fecal calprotectin measurements to detect endoscopic recurrence in postoperative Crohn's disease: is colonoscopic surveillance no longer needed? Gastroenterology. 2015;148(5):889-92.

24 Boschetti G, Laidet M, Moussata D, Stefanescu C, Roblin X, Phelip G, et al. Levels of fecal calprotectin are associated with the severity of postoperative endoscopic recurrence in asymptomatic patients with Crohn's disease. Am J Gastroenterol. 2015;110(6):865-72.
25 Wright EK, Kamm MA, De Cruz P, Hamilton AL, Ritchie KJ, Krejany EO, et al. Measurement of fecal calprotectin improves monitoring and detection of recurrence of Crohn's disease after surgery. Gastroenterology. 2015; 148(5):938-e1.

26 Qiu Y, Mao R, Chen BL, He Y, Zeng ZR, Xue $\mathrm{L}$, et al. Fecal calprotectin for evaluating postoperative recurrence of Crohn's disease: a meta-analysis of prospective studies. Inflamm Bowel Dis. 2015;21(2):315-22.

27 Sailer J, Peloschek P, Reinisch W, Vogelsang $\mathrm{H}$, Turetschek K, Schima W. Anastomotic recurrence of Crohn's disease after ileocolic resection: comparison of MR enteroclysis with endoscopy. Eur Radiol. 2008;18(11):251221.

28 Aberra FN, Lewis JD, Hass D, Rombeau JL, Osborne B, Lichtenstein GR. Corticosteroids and immunomodulators: postoperative infectious complication risk in inflammatory bowel disease patients. Gastroenterology. 2003;125(2):320-7.

29 Tay GS, Binion DG, Eastwood D, Otterson MF. Multivariate analysis suggests improved perioperative outcome in Crohn's disease patients receiving immunomodulator therapy after segmental resection and/or strictureplasty. Surgery. 2003;134(4):565-3; discussion 72-3.

30 Chang MI, Cohen BL, Greenstein AJ. A review of the impact of biologics on surgical complications in Crohn's disease. Inflamm Bowel Dis. 2015;21(6):1472-7.

31 Kopylov U, Ben-Horin S, Zmora O, Eliakim R, Katz LH. Anti-tumor necrosis factor and postoperative complications in Crohn's disease: systematic review and meta-analysis. Inflamm Bowel Dis. 2012;18(12):2404-13

32 Shelton SK, Chukwulebe SB, Gaieski DF, Abella BS, Carr BG, Perman SM. Validation of an ICD code for accurately identifying emergency department patients who suffer an out-of-hospital cardiac arrest. Resuscitation. 2018 Jan 13;125:8-11. Epub ahead of print. 